\title{
Surgical endodontic retreatment success and failure are almost equivalent
}

\author{
In patients with radiographically-determined failure of surgical endodontics, \\ what is the success rate of repeat surgery?
}

\begin{abstract}
Peterson J, Gutmann JL. The outcome of endodontic resurgery: a systematic review. Int Endod J 2001; 34:169-175
\end{abstract}

Data sources Medline, EMbase, Science Citation Index, US National Library of Medicine, along with searches made on the Internet.

Study selection Studies were included if they: had a random research design; were peer-reviewed; follow-up was at least 1 year; and if retrofilling material, the healing group, age, sex and teeth/roots, the number of surgeons, and the age and sex of patients were identified.

Data extraction and synthesis The outcome in each of the included studies was standardised to success, or to uncertain or unsuccessful outcome, and the weighted average was calculated.

Results Eight eligible studies were identified, involving 2375 patients and 2788 teeth. The weighted average for success with initial surgery was $64.2 \%$ with $25.7 \%$ uncertain and $15.75 \%$ unsuccessful. For patients undergoing a second surgery the weighted average success rate was $35.7 \%$ with $26.3 \%$ uncertain and $38 \%$ unsuccessful.

Conclusions In patients who underwent second surgery for radiographically-determined endodontic failure, $35.7 \%$ healed successfully.

\section{Commentary}

When teeth have irreversible pulp disease the best course of action is root canal treatment. Conventional root canal treatment aims to remove the micro-organisms and pulp tissue from the canal system and repair the tooth by filling the canal system and restoring the lost coronal tissue. In this way, the surface integrity of the tooth is restored and the barrier to microbial ingress re-established. Unfortunately, the technical difficulties inherent in root canal treatment occasionally results in micro-organisms remaining within the canal system and, thus, continuation of the disease process. In most cases conventional retreatment of failed cases is indicated ${ }^{1}$ because this is the most appropriate way of ensuring the effective removal of any remaining micro-organisms. On occasion, however, surgical endodontics in the form of root-end resection and root-end filling are preferred. The success rate of surgery has been reported to range from $25 \%$ to $99 \%{ }^{2}$ with the result that, in failed cases, the clinician has to decide whether to attempt a second surgical procedure, a resurgery, or whether to attempt an alternative and more predictable strategy for removing micro-organisms, e.g., extraction. Data on the outcome of resurgery are sparse.

The aim of this paper is to establish an outcome standard for the assessment of healing radiographically after resurgery for persistent periradicular lesions by systematically reviewing the results from published studies. It did not include clinical parameters. An extensive literature search was undertaken and 42 papers qualified for the inclusion initially, with eight papers reporting resurgery results from the period 1970 to 1997 . The inclusion eligibility criteria for the systematic review were not met by all eight reports, but these deficiencies were identified by the authors as being less important than the diminished overall sample population for the review if they had been excluded.

This review highlights the limited data available about resurgery, the limited post-operative follow-up periods reported and other deficiencies in reporting that often exclude otherwise valuable studies from further consideration. The review also highlights the low success rate for resurgery in the period 1970-1997 and the limited value of amalgam as a root-end material. Overall, the systematic review fulfils its aim of providing a baseline outcome standard for resurgery.

\section{Practice points}

- As a result of a greater understanding of the disease process it is clear that micro-organisms remaining in the root canal system should be removed wherever possible using conventional root canal treatment.

- The use of micro-surgical techniques, magnification and new materials such as mineral trioxide aggregate can enhance the success rate of initial surgery. ${ }^{3}$

- It can be anticipated that the outcome of resurgery using such techniques would be higher than those derived from the systematic review.

\section{Paul MH Dummer}

Department of Adult Dental Health, Dental School, University of Wales College of Medicine, Cardiff, Wales, UK

1. Reit C. On decision making in endodontics. A study of diagnosis and management of periapical lesions in endodontically treated teeth. Swedish Dent J 1986; 41(Suppl.):S1-S30.

2. Gutmann JL, Harrison JW. Surgical Endodontics. St Louis: Ishiyaku EuroAmerica.

3. von Arx T, Gerber C, Hardt N. Periradicular surgery of molars: a prospective clinical study with a one-year follow-up. Int Endod J 2001; 34:520-525.

Evidence-Based Dentistry (2003) 4, 51.

doi:10.1038/sj.ebd.6400192 